Connotas. Revista de crítica y teoría literarias 
CONNOTAS. REVISTA DE CRÍTICA Y TEORÍA LITERARIAS. Enero - diciembre 2013, es una publicación anual editada por la Universidad de Sonora, a través de la División de Humanidades y Bellas Artes, en el Departamento de Letras y Lingüística. Blvd. Luis Encinas y Blvd. Rosales s/n, Col. Centro, C.P. 83000, Hermosillo Sonora; Tel. (662) 2592 136, (662) 2592 157, <www.uson.mx>, <http://www.connotas.uson.mx, connotas@capomo.uson.mx>. Editor responsable: Rosario Fortino Corral Rodríguez. Reservas de Derechos al Uso Exclusivo núm. 04-2006-020714184900-102. ISSN: 18706630; ambos otorgados por el Instituto Nacional del Derecho de Autor. Licitud de Título núm. 13434 y de Contenido núm. 11007, otorgados por la Comisión Calificadora de Publicaciones y Revistas Ilustradas de la Secretaría de Gobernación. Este libro se publicó con el apoyo del Programa Integral de Fortalecimiento Institucional C/PIFI 2013-12934

Se autoriza la reproducción total o parcial de los contenidos de la presente publicación, siempre y cuando se acredite adecuadamente el origen de los mismos.

Datos de contacto para la publicación: División de Humanidades y Bellas Artes, Departamento de Letras y Lingüística; Apartado Postal 793, Col. Centro, C.P. 83000, Hermosillo, Sonora, México. Tels.: (662) 259-21-87, Tel-fax 212-55-29. Correo electrónico: connotas@capomo.uson.mx Página web: http://www.connotas.uson.mx 


\section{Connotas. Revista de crítica y teoría literarias}

Núm. 13, año 2013

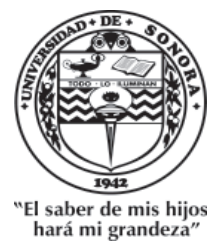


UNIVERSIDAD DE SONORA

RECTOR

Heriberto Grijalva Monteverde

VICERRECTORA

Arminda Guadalupe García de León Peñúñuri

Secretario General Académico

Enrique Fernando Velázquez Contreras

División de Humanidades y Bellas Artes

María Rita Plancarte Martínez

Departamento de Letras y LingÜística

Ana Bertha de la Vara Estrada

César Avilés Icedo

Rosa María Burrola Encinas

Fortino Corral Rodríguez

Leticia Martínez Figueroa

Jesús Abad Navarro Gálvez

Gabriel Osuna Osuna

María Rita Plancarte Martínez

DiRECTOR

Fortino Corral Rodríguez

Giuseppe Bellini

Consejo InTERnacional

Universidad de Milán

Carlos Pacheco

Luis Beltrán Almería

Universidad Simón Bolivar

Universidad de Zaragoza

Rafael Olea Franco

Helena Beristáin

El Colegio de México

Joan Oleza Simó

Universidad Nacional Autónoma de México

Universidad de Valencia

Raúl Bueno-Chávez

Dartmouth College

Julio Ortega

Evodio Escalante

Brown University

Luz Aurora Pimentel

Universidad Autónoma Metropolitana

Universidad Nacional Autónoma de México

Beatriz González-Stephan

Susana Reisz

Rice University

Aníbal González

The City University of New York.

José Carlos Rovira

Universidad de Alicante

Yale University

Aurelio González Pérez

Charles Tatum

El Colegio de México

Yvette Jiménez de Báez

The University of Arizona

Jorge Urrutia

El Colegio de México

Universidad Carlos III de Madrid

Nelson Osorio Tejeda

Universidad de Santiago de Chile

Emil Volek

Arizona State University 


\section{Índice}

\section{Artículos}

Una hermenéutica analógica para la literatura

Mauricio Beuchot Puente

La obra de Jorge Luis Borges durante la década de 1930: estudio de algunos elementos para la conformación de su poética en Discusión (1932)

Daniel Zavala Medina

Metáforas de la locura y la muerte en "Río subterráneo" de Inés Arredondo

Gabriel Osuna Osuna

La alteridad como vector de la construcción del yo: La muerte me da de Cristina Rivera Garza y El Gran Vidrio de Mario Bellatin Véronique Pitois Pallares

Uno soñaba que era rey de Enrique Serna: un espacio de contradicción Claudia Gidi

Una angustia compartida. Análisis de la voz narrativa en Mi hermano Carlos de Jorge López Páez

Jorge Antonio Muñoz Figueroa

De Los perros románticos y otros poemas de Roberto Bolaño Marina Martínez Andrade 
Las novelas del Crack, multiplicidad y superposición de mundos Ramón Alvarado Ruiz

La construcción del “otro" en la Relación de la jornada de Cíbola de Pedro Castañeda de Nájera

Guillermo Martínez Sotelo

Destino e historia en Margarita, está linda la mar de Sergio Ramírez

María Rita Plancarte Martínez

\section{Notas}

Identidad negada y mundo al revés en Santa María del Circo de David Toscana

César Avilés Icedo y María Elena González Borgaro 203

"El buen ejemplo" de Vicente Riva Palacio: la historia de un cuento

Marco Antonio Chavarín González

Organización descriptiva en Catarina de San Juan, princesa de la India y visionaria de Puebla

Silvia Guadalupe Alarcón Sánchez

Genealogías en Todo aqui es polvo de Esther Seligson. Lectura a partir de los epígrafes

Luz Elena Zamudio Rodríguez

\section{Reseñas}

Martha Elena Munguía Zatarain. La risa en la literatura mexicana (apuntes de poética)

Silvia Alicia Manzanilla Sosa 
Véronique Pitois-Pallares. El arte del fragmento: El Gran Vidrio de Mario Bellatin

Julio César Zárate Ramírez 255

Nicolás Kanellos. Hispanic Immigrant Literature: El sueño del retorno Josué Gutiérrez González

Dante Salgado. Brevísima relación de la idea de amor en Occidente Diana Vanessa Geraldo Camacho 271

Mara L. García. Paco Yunque: una mirada crítica Juan Paredes Carbonell 279

Abstracts 283

Résumés

Normas editoriales 



\title{
La obra de Jorge Luis Borges durante la década de 1930: estudio de algunos elementos para la conformación de su poética en Discusión (1932)
}

\author{
Daniel Zavala Medina*
}

\section{Resumen:}

En este ensayo se examinan algunas características de la obra de Jorge Luis Borges durante la década de 1930. Una de mis hipótesis de trabajo es que hubiera sido imposible la concepción de la obra que produjo a partir de los años cuarenta, sin el periodo de preparación estético que representó la década previa. En particular, la lectura de un libro como Discusión permite identificar algunos de los elementos que se volvieron esenciales para el argentino a partir de sus primeras colecciones de cuentos; también, varios de los aspectos estético-literarios predominantes en la Argentina de la época, y de los cuales iría tomando distancia paulatinamente.

Palabras clave:

Jorge Luis Borges, Discusión, "El arte narrativo y la magia", Poética.

Es extraordinariamente complejo el retrato literario que podría trazarse del denominado "primer Borges" (Olea Franco, El otro Borges; Louis; Sarlo, Borges, un escritor en las orillas; Sarlo y Altamirano, Ensayos; Montaldo). Después del regreso familiar de su estancia europea y a

* Universidad Autónoma de San Luis Potosí. 
partir de 1921, él participó con profusión y constancia en el mundo de las letras de Buenos Aires. Su legado consistió en un notable y arduo recorrido a través de varias estaciones de tránsito: vanguardismo ultraísta; nacionalismo criollista; tres libros de poesía; tres libros de ensayos que después se empeñaría en condenar al olvido... No obstante, los especialistas coinciden en que, hacia finales de los años veinte y principios de los treinta del siglo XX, el joven Borges vivió un periodo de crisis creativa. Una de las consecuencias más visibles de esa crisis fue un alejamiento temporal de la creación poética (Pastormelo 60-63, 130-135).

Hay un reconocimiento unánime de que la obra narrativa, ensayística y poética del escritor argentino Jorge Luis Borges (18991986) es una de las más importantes de la literatura hispanoamericana del siglo XX. La primera obra fundamental de su carrera fue, quizás, El jardín de senderos que se bifurcan (1941). A ésta seguirían libros de cuentos como Ficciones (1944) (en el cual se reunieron los ocho relatos de El jardin... y seis de una nueva sección: Artificios) y El Aleph (1949). Es decir, fue a partir de los años cuarenta que la trayectoria de Borges llegó a los niveles de creatividad y complejidad que lo han hecho famoso a nivel internacional.

Sin embargo, si el genio argentino escribía poesía durante los años veinte y vivió una crisis creativa a finales de esa década, y llegó a la cúspide de su carrera durante los cuarenta, ¿qué ocurrió en los años de 1930? En este ensayo se pretende examinar algunas características de la obra de Jorge Luis Borges durante esa década. Una de mis hipótesis de trabajo es que hubiera sido imposible la concepción de las tres colecciones de cuentos mencionadas arriba -o de la Antología de la literatura fantástica (1940), reunida en colaboración con Adolfo Bioy Casares y Silvina Ocampo- sin el periodo de preparación estético que representó la década de 1930. A pesar de ello, más allá de El otro Borges. Elprimer Borges de Rafael Olea Franco o de Jorge Luis Borges: cuvre et mancuvres de Annick Louis, aún no parece suficiente lo que se ha investigado al respecto.

Una de las obras que, a mi juicio, es imprescindible estudiar es el libro de ensayos Discusión (1932). Desde mi perspectiva, algunos de los trabajos incluidos en este volumen son fundamentales en el desa- 
rrollo de la futura poética borgeana. Me refiero, en principio, a textos como "La postulación de la realidad" y "El arte narrativo y la magia", en los cuales el autor toma distancia con respecto a la estética narrativa predominante durante esa época: el realismo. Por otro lado, conviene analizar otros ensayos como "El coronel Ascasubi", "El Martín Fierro" y "Nuestras imposibilidades", donde Borges cuestionará otro tópico contemporáneo que había marcado su propia obra en la década de 1920: la veta criollista-nacionalista.

De esta manera, la lectura atenta de un libro como Discusión permitiría identificar algunos de los elementos que se volvieron esenciales para el argentino a partir de sus primeras colecciones de cuentos; también, varios de los aspectos estético-literarios predominantes en la Argentina de la época, y de los cuales iría tomando distancia paulatinamente.

Un ensayo de Discusión que considero central es "El arte narrativo y la magia". Este trabajo fue publicado originalmente en la revista Sur (Borges, "El arte narrativo y la magia"). Espacio escogido de manera premeditada: Beatriz Sarlo ha estudiado en un trabajo ya clásico las estrategias desplegadas tras esta elección (Sarlo, "Borges en Sur").

En la primera línea de "El arte narrativo y la magia" se lee: "El análisis de los procedimientos de la novela ha conocido escasa publicidad" (Borges Discusión 109). Así, en tanto que una de las preocupaciones fundamentales en el ensayo es el examen de los procedimientos de la novela, se sobreentiende que lo que Borges procurará dilucidar son los problemas básicos en la construcción de una arquitectura novelística.

Las obras que le sirven al argentino para exponer sus convicciones son The Life and Death of Jason (1867) de William Morris y Narrative of Arthur Gordon Pym (1838) de Edgar Allan Poe. Hay hilos de continuidad entre este ensayo y "La postulación de la realidad", también incluido en Discusión: en éste, Borges había citado un largo pasaje de la novela de Morris y lo había celebrado como ejemplo de un arte clásico (96-97).

Ahora bien, la elección de ese par de volúmenes es, a todas luces, sorprendente: ¿por qué prescindir en una meditación sobre la novelística, justamente, de los clásicos decimonónicos europeos?; ¿ ¿por 
qué anteponer a los maestros ingleses, franceses y rusos un libro en verso de corte mitológico o una novela que es única en las obras completas del norteamericano (y que, para los parámetros clásicos, difícilmente se clasificaría como una novela)? Emir Rodríguez Monegal explica: "En las dos obras hay un elemento común: el rechazo tácito de la "poética" del realismo" (179). ${ }^{1}$

Borges expone primeramente The Life and Death of Jason:

El proyecto de Morris era de casi imperceptible, íntima valentía. Era la relación auténtica de las aventuras apócrifas de Jasón, rey de Iolcos. La sorpresa lineal, recurso general de la lírica, no era posible en esa narración de más de diez mil versos. Ésta necesitaba ante todo una fuerte apariencia de veracidad, si no absoluta, capaz a lo menos de producir esa espontánea suspensión de la duda, que determina para Coleridge la fe poética. Morris consigue despertar esa fe; quiero investigar cómo. (Discusión 110)

Creo que hay dos elementos definitivos en la elección de The Life and Death of Jason como raíz de la argumentación borgeana. Cuando se enfatiza que la mayor dificultad de Morris era conseguir "la relación auténtica de las aventuras apócrifas de Jasón”, se nos ubica ante el problema de la verosimilitud. ${ }^{2}$ Es decir, se nos pone delante de una

${ }^{1}$ La tesis de este estudio es, de hecho, que "El arte narrativo y la magia" es "un ataque a fondo del realismo" (Rodríguez Monegal 177).

2 Annick Louis indica: "Dans le système de Borges le problème de la vraisemblance apparait comme une question strictement littéraire n'ayant aucun rapport avec les allusions à des objets, à des faits ou à des personnages réels. Ce qui est vraisemblable l'est en fonction de la constitution de traits circonstanciels de la narration; il est inutile pour l'écrivain de se documenter ou de fournir une quantité importante de détails. Borges pousse le refus du réalisme et du naturalisme, deux esthétiques très en vogue dans l'Argentine de l'époque, à un point extrême, en suggérant que les romans qui répondent à celles-ci sont peu vraisemblables. Chaque écrivain se doit donc de créer un système de vraisemblance dans ses fictions; un système ayant des lois et une logique propres" (163). 
de las mayores preocupaciones de casi cualquier representación en la literatura. Borges, sin embargo, la emplea para cuestionar -aquí tácitamente; más delante de forma directa- a las corrientes vinculadas con el realismo: ¿son las técnicas realistas la solución óptima cuando se busca la representación del mundo en la literatura?

El segundo de los puntos trascendentes en la selección de la "novela" de Morris es, justamente, el debate sobre la novela como género. ¿Podemos hablar de la obra de William Morris como una novela? Para Beatriz Sarlo, el corpus aducido en "El arte narrativo y la magia", sólo podría llamarse novelístico con grandes reservas ("Borges en Sur" 5). No obstante, la lectura de otros ensayos de Discusión permite establecer conexiones argumentales al interior del libro para salvar esta cuestión.

En "El Martín Fierro", luego de comentar varios pasajes de ese poema, Borges ofrece sus conclusiones: "En esta discusión de episodios me interesa menos la imposición de una determinada tesis, que este convencimiento central: la esencia novelística del Martín Fierro, hasta en los pormenores. Novela, de organización cuidada o genial, es nuestro Martín Fierro..." (Discusión 60). La obra capital de la literatura argentina no sería, entonces, un poema épico sino una novela: lo privativo novelístico no radica, por lo tanto, en el uso de la prosa sino en el de los procedimientos narrativos. Además, y aunque aquí no lo diga de manera explícita, para Borges el Martín Fierro también es una novela por la certera construcción literaria de dos de sus protagonistas: Fierro y Cruz.

En la última nota explicativa de "El Martín Fierro" se procura justificar la versificación de la "novela" de José Hernández:

Queda su condición de verso. Por ella ha trascendido a las guitarras y a los hombres de la distancia; a ella se debe enteramente su difusión oral. Tiene además otra recelada virtud, que se refiere a la economía interna del libro, no a la mecánica de su éxito. El verso - como el coturno, la veneración y la máscara, en la tragedia esquílea- concede al Martín Fierro ese grado mínimo de irrealidad, que es condición del arte. Una directa narración -recuérdese el problema de Güiraldes, en Don Segun- 
do Sombra- demandaría un interlocutor, un motivo. El verso, en cambio, se presenta y está. (Discusión 64)

Son varias las materias que convergen en este párrafo. El uso del verso zanjaría por lo menos tres cuestiones. Primeramente, a través de la versificación se explican, por un lado, su economía de recursos; por el otro, las posibilidades de su transmisión oral $-\mathrm{y}$ parte de su éxito- se basan en el metro y en la rima como apoyos mnemónicos. En segundo lugar, Borges asegura que el Martín Fierro es una novela, pero ésta no tendría vínculos con el realismo: la idea de que "la irrealidad es una condición del arte" es, sin lugar a dudas, parte de su programa estético. Esta poética fue defendida por Borges en otros textos y durante varios años. ${ }^{3}$ Finalmente, el verso -de acuerdo con Borges- permitiría franquear el escollo de la necesidad de un interlocutor y de un motivo que legitime la narración. Es muy impresionante, desde luego, la energía de la frase "El verso, en cambio, se presenta y está": aquí no parece que se argumente; se espeta.

Prolongaré la digresión en mi lectura de "El arte narrativo y la magia". Puesto en contexto, defender la tesis de que el Martín Fierro es una novela era mucho más que una simple extravagancia. En este aparente desvarío subyace una vieja batalla literaria. A raíz del centenario del inicio del movimiento independentista argentino, de la numerosa presencia de inmigrantes en el país, de las características de la lengua local frente a los idiomas foráneos y del cuestionamiento acerca de las relaciones que debía establecer el país con el resto de los estados, hubo un movimiento de reflexión en torno a la identidad nacional (Olea Franco, El otro Borges; Louis; Sarlo, Borges, un escritor en las orillas; Sarlo y Altamirano, Ensayos; Montaldo). Algunos de estos temas controversiales cristalizaron en una compleja revaloración del

${ }^{3}$ Ahora citaré sólo un ejemplo, correspondiente a la década posterior. En el relato "El milagro secreto" (publicado originalmente en la revista Sur, en 1943), leemos: "[Jaromir] Hladik preconizaba el verso [para su drama Los enemigos], porque impide que los espectadores olviden la irrealidad, que es la condición del arte" (Borges "El milagro" 16). 
Martín Fierro: "La nueva lectura del poema de Hernández no sólo fue ocasión para la transfiguración del gaucho -convertido en arquetipo de la raza-, sino también para establecer un texto 'fundador' de la nacionalidad" (Sarlo y Altamirano Ensayos 186-187).

Sarlo y Altamirano explican que esa corriente de revaloración del Martín Fierro tuvo varios episodios claves: las conferencias dictadas por Leopoldo Lugones - a las cuales asistió la oligarquía argentina en pleno, incluido Roque Sáenz Peña, presidente de la república-, recogidas después en El payador (1916); el discurso que pronunció Ricardo Rojas cuando tomó a su cargo la recién fundada cátedra de Literatura Argentina; y, por último, la controversia promovida por los editores de la revista Nosotros, con una encuesta en torno al Martín Fierro (Ensayos 187). El sondeo de Nosotros tenía por tema la naturaleza épica del poema hernandiano: Lugones y Rojas la habían afirmado, "aunque le asignaran una filiación histórico-literaria diferente. Para el primero, el Martín Fierro tenía sus antepasados en los poemas homéricos, mientras que para Rojas se emparentaba con la épica medieval: la obra de Hernández representaba para los argentinos lo que la Chanson de Roland para los franceses y el Cantar de Mio Cid para los españoles" (187).

Beatriz Sarlo asegura que "quien escribiera en la Argentina del primer tercio [del siglo $\mathrm{XX}$ ] tenía que examinar el mito gaucho y medirse con él, ya fuera para rechazarlo, para desviarlo o para adoptarlo" (Sarlo Borges, un escritor en las orillas 61-62). Esto es, justamente, lo que hace Borges cuando reivindica la naturaleza novelística del poema. ${ }^{5}$ Escribe en el ensayo de Discusión:

Se me recordará que las epopeyas antiguas representan una preforma de la novela. De acuerdo, pero asimilar el libro de Hernández a esa categoría primitiva es agotarse inútilmente en

${ }^{4}$ Aunque algunos han puesto en duda la presencia del primer mandatario argentino (Dobry 17, 185).

${ }^{5}$ Borges reiteraría esta posición en otras ocasiones. Por ejemplo, como respuesta a la "Encuesta sobre la novela" que convocó la Gaceta de Buenos Aires el 6 de octubre de 1934 (Textos recobrados. 1931-1955, 346-347). 
un juego de fingir coincidencias, y es renunciar a toda posibilidad de un examen. La legislación de la épica -metros heroicos, manejo servicial de los dioses, destacada situación política de héroes- no es aplicable aquí. Las condiciones novelísticas, sí lo son. (Discusión 60-61)

Con este solo aserto, Borges arrebató el Martín Fierro a Lugones y a Rojas: las connotaciones míticas quedaban, así, difuminadas. Ahora bien, hay que señalar que si bien procuró alejar el poema de las disputas en torno a la nacionalidad argentina, Borges intentó imponer -con notable éxito- su propia lectura del Martín Fierro a lo largo de varias décadas. Basta pensar en "El fin" como un relato con el que buscó clausurar el poema gauchesco, contraviniendo incluso algunas de las intenciones de Hernández - que Borges juzgó reaccionarias- en la segunda parte de la obra (Louis 427-443; Sarlo Borges, un escritor en las orillas 60-67; Olea Franco "El íntimo"; García).

Vuelvo ahora a "El arte narrativo y la magia". Hay hilos de continuidad entre éste y "La postulación de la realidad" cuando se analizan las estrategias para conseguir la verosimilitud en The Life and Death of Jason. Borges recuerda la presentación de un par de seres mitológicos: Quirón y las sirenas. En esas presentaciones identificamos el tercero de los recursos para postular la realidad: la invención circunstancial. En el caso del centauro, una inesperada "corona de hojas de encina en la transición de bruto a persona" (Borges Discusión 112); en el de las sirenas, "la neblina ansiosa de lágrimas que ofusca la visión de los hombres" (113), cuando descubren a las mujeres míticas.

Al finalizar el examen de la novela de Morris, el ensayista pasa a Narrative of Arthur Gordon Pym. Abajo destacaré los temas pertinentes para este trabajo en el relato de Poe. Ahora prefiero estudiar la conclusión borgeana que se desprende del análisis de las novelas:

Rectamente se induce de lo anterior que el problema central de la novelística es la causalidad. Una de las variedades del género, la morosa novela de caracteres, finge o dispone una concatenación de motivos que se proponen no diferir de los del mundo real. Su caso, sin embargo, no es el común. En la 
novela tumultuosa y en marcha, esa motivación es improcedente, y lo mismo en el relato de breves páginas y en la infinita novela espectacular que compone Hollywood con los plateados ídola de Joan Crawford y que las ciudades releen. Un orden muy diverso los rige, lúcido y ancestral. La primitiva claridad de la magia. (Discusión 117)

Como apuntamos, la primera línea de "El arte narrativo y la magia" plantea una inquietud básica: los fundamentos de la construcción de una novela. El corolario es que la arquitectura novelística tiene como cimiento la causalidad. ${ }^{6}$ A partir de esta conclusión, postula una clasificación dual e incompatible de novelas: la que aquí denomina "novela de caracteres" -marcada por su intencionalidad realista- y la novela construida con base en una "causalidad mágica".

Ahora bien, en tanto que Borges se interroga sobre los procedimientos de la novela y concluye que el carácter medular de ésta radica en la causalidad, se originan dos consecuencias complementarias. Por un lado, está colocando en un primer plano cuestiones de tipo constructivo y formal. Por el otro, desplaza prácticamente toda preocupación por el contenido de la novela: poco importa qué se narre, lo que interesa es qué solución formal se utilice para hacerlo.

Esta posición estética era extraordinariamente subversiva en su momento. Cuando Borges escribió estas consideraciones, uno de los elementos clave que organizaban el sistema literario argentino era una novela afín al realismo. Ir a contrapelo de esta corriente literaria tenía serias consecuencias: no sólo era rechazar un modelo de representación literario; también implicaba marcar distancia con respecto de las preocupaciones éticas que, para algunos narradores argentinos, debían definir su contenido.

${ }^{6}$ Véase la reseña, casi contemporánea a Discusión, de la novela 45 días y treinta marineros de Norah Lange, en la cual se analiza la obra desde esta misma perspectiva (Revista Multicolor de los Sábados del diario Crítica -9 de diciembre de 1933) (Borges. Textos recobrados. 1931-1955, 77-78). Véase también Louis (163). 
El realismo no sólo dominaba el sistema literario de la nación sudamericana durante las décadas de 1920-1930: su presencia era notoria a nivel continental (Rama). Beatriz Sarlo sintetiza la situación del momento al que nos referimos: "la literatura argentina de esos años mantiene una relación, si se quiere tardía, con las poéticas del realismo, el costumbrismo y la novela psicológica de personaje. Borges se separa drásticamente de las poéticas del realismo que incluso habían marcado, como en los cuentos de Quiroga, a la literatura fantástica" ("Una poética" 21). Sólo hay un punto que habría que matizar: no es que la literatura fantástica de Horacio Quiroga esté señalada por la poética realista; más bien él practicó una obra fantástica de corte clásico, la cual -como postuló Tzvetan Todorov- implicaba por necesidad un contexto afín con el realismo.

Por otro lado, el mapa literario que dibuja Sarlo de ese periodo es el siguiente:

El realismo forma parte de la otra literatura en la Argentina: naturalismo a lo Manuel Gálvez (que la vanguardia martinfierrista había pulverizado sin necesitar otro recurso que la ironía), moralismo pequeñoburgués de la literatura humanitarista de Boedo escrita por Leónidas Barletta o Álvaro Yunque, pintoresquismo porteño a lo Enrique González Tuñón, naturalismo negro y teratológico, como el de Elías Castelnouvo, fascinado por los monstruos producidos en una sociedad de clases. (Sarlo "Una poética" 21)

Graciela Montaldo destaca el compromiso ético que una literatura como la arriba trazada debía desempeñar: "con la literatura había que fortalecer la nacionalidad americana, educar a las masas, moralizar la sociedad y por esta razón, los textos producidos van a ser generalmente tributarios de ideas extra-literarias, de las que se muestran como ejemplos" (227). Si admitiéramos que el problema central de la novelística es la causalidad, estaríamos suscribiendo una poética que tiene muy poco que ver con las preocupaciones morales. En "Paul Groussac", otro de los ensayos de Discusión, Borges declara: "Soy un lector hedónico: jamás consentí que mi sentido del deber 
interviniera en afición tan personal como la adquisición de libros..." (125). ¿Qué mayor escándalo que declararse un lector -e, implícitamente, un escritor- hedonista en un periodo convulsionado por los conflictos, en un mundo que requería de un mínimo compromiso con la realidad? La mayoría de las acusaciones contra Borges por su falta de contenido humano podrían explicarse por una concepción de la literatura de este tipo (Bastos).

Cuando Borges comenta la Narrative of Arthur Gordon Pym, en "El arte narrativo y la magia", declara: "El secreto argumento de esa novela es el temor y la vilificación de lo blanco" (Discusión 114). En esta observación me parece que subyace, precisamente, un apunte acerca de las complejidades morales. En la cultura occidental moderna, suele considerarse el blanco como el color del bien y de la pureza. Sin embargo, Borges examina la novela de Poe y recuerda, también, Moby Dick como obras donde la blancura simboliza el mal. Con esto, creo que lanza una especie de crítica contra posturas literarias maniqueas, que pretenden la sanción de graves conflictos éticos en el mundo real.

Concluyo con "El arte narrativo y la magia". Una vez que Borges ha tomado partido por la causalidad mágica como el fundamento de una construcción novelística ideal, explica el mecanismo secreto de la magia:

Ese procedimiento o ambición de los antiguos hombres ha sido sujetado por Frazer a una conveniente ley general, la de la simpatía, que postula un vínculo inevitable entre cosas distantes, ya porque su figura es igual -magia imitativa, homeopáticaya por el hecho de una cercanía anterior -magia contagiosa. Ilustración de la segunda era el ungüento curativo de Kenelm Digby, que se aplicaba no a la vendada herida sino al acero delincuente que la infirió -mientras aquélla, sin el rigor de bárbaras curaciones, iba cicatrizando. De la primera los ejemplos son infinitos. (Discusión 117-118)

El ensayista ubica uno de esos infinitos ejemplos entre los hechiceros de Australia Central, quienes "se infieren una herida en el ante- 
brazo que hace correr la sangre, para que el cielo imitativo o coherente se desangre en lluvia también" (Discusión 118). ${ }^{7}$ La aproximación prodigiosa de acontecimientos distantes, la influencia mutua e insospechada de los fenómenos más remotos, es precisamente lo que Borges recupera de la causalidad mágica para proyectarlo como uno de los paradigmas de la construcción novelística: "Ese recelo de que un hecho temible pueda ser atraído por su mención, es impertinente o inútil en el asiático desorden del mundo real, no así en una novela, que debe ser un juego preciso de vigilancias, ecos y afinidades. Todo episodio, en un cuidadoso relato, es de proyección ulterior" (119-120). ${ }^{8}$

Para Borges, uno de los mayores artífices de este tipo de construcción literaria es Gilbert Keith Chesterton, y comenta varios ejemplos de cómo procede el escritor inglés, lo cual nos lleva a uno de los géneros con los cuales comenzó a experimentar en la década de 1930: el policial clásico. Esta arquitectura ideal, sin embargo, no se limita a la página escrita: "Esa teleología de palabras y de episodios es omnipresente también en los buenos films" (121). En todos los ejemplos convocados por el argentino se subraya el modo en el cual los creadores diestros logran que las escenas iniciales sean un sutil e insospechado anticipo de la conclusión de la obra.

${ }^{7}$ La recuperación de una olvidada reseña permite observar que la curiosidad borgeana por la "causalidad mágica" se estaba gestando desde varios años antes, hacia mediados de la década de 1920. El libro reseñado se titula Cuentos del Turquestán, y la nota apareció originalmente en el diario La Prensa de Buenos Aires -29 de agosto de 1926-, bajo la firma de "Luis Borges". El joven comenta sobre esos relatos orientales: "La magia es un episodio causal, es ejemplo de causalidad como tantos otros. Para matar de lejos a un hombre, podemos atravesarlo a flechazos; pero también podemos amasar un monigote suyo de arcilla y clavetearlo hasta que se muera el original. [...] Hablamos de leyes naturales, y todo lector de Eduardo Carpenter o de Ernesto Mach sabe que esas leyes no pasan de provechosas ficciones y que se las inventamos al mundo. El hombre primitivo no cree ni en esas prefijadas leyes ni en el milagro que es su bienhechora infracción y que las precisa para vivir con algún decoro. Cree instintivamente en la causalidad y no la clasifica en mágica y en común" (Textos recobrados. 1919-1929, 262).

${ }^{8}$ Las cursivas son mías. 
No creo que sea fortuito que el ensayista utilice la palabra "teleología" en su análisis de las construcciones artísticas. Si a Borges le interesa un tipo de literatura -y de cinematografía- que logre dar coherencia a todos y cada de los episodios presentados -y que en su exposición inicial podrían parecer casuales o de justificación endeble-, es natural que use un término ligado a la noción de un fin último, el cual conseguiría justificar todo el desarrollo.

En el último párrafo de "El arte narrativo y la magia" se lee: "Procuro resumir lo anterior. He distinguido dos procesos causales: el natural, que es el resultado incesante de incontrolables e infinitas operaciones; el mágico, donde profetizan los pormenores, lúcido y limitado. En la novela, pienso que la única posible honradez está en el segundo. Quede el primero para la simulación psicológica" (121122). Si nos quedara alguna duda de este ensayo como estandarte en contra de la novela realista, creo que estas líneas del ensayo definitivamente las despejarían.

\section{Bibliografía}

Bastos, María Luisa. Borges ante la crítica argentina. 1923-1960. Buenos Aires: Hyspamerica, 1974.

Borges, Jorge Luis. Discusión. Buenos Aires: Gleizer, 1932. . "El arte narrativo y la magia". Sur 5 (1932): 172-179. "El milagro secreto". Sur 101 (1943): 13-20. Textos recobrados. 1919-1929. Buenos Aires: Emecé, 1997. Textos recobrados. 1931-1955. Buenos Aires: Emecé, 2001.

Dobry, Edgardo. Una profecía del pasado. Lugones y la invención del "linaje de Hércules". Buenos Aires: FCE, 2010.

García Morales, Alfonso. "Jorge Luis Borges, autor del Martín Fierro". Variaciones Borges 10 (2000): 29-64.

Louis, Annick. Jorge Luis Borges: eure et mancwvres. Paris: L'Harmattan, 1997.

Montaldo, Graciela. "Borges: una vanguardia criolla". Historia social de la literatura argentina. Yrigoyen entre Borges y Arlt. Ed. Graciela Montaldo. Vol. VII. Buenos Aires: Contrapunto, 1989. 213-232. 
Olea Franco, Rafael. "El íntimo cuchillo en la garganta: ¿civilización o barbarie?”. Olea Franco, Rafael. Los dones literarios de Borges. Madrid: Iberoamericana, 2006. 37-66.

. El otro Borges. El primer Borges. Buenos Aires: El Colegio de México-FCE, 1993.

Pastormelo, Sergio. Borges crítico. Buenos Aires: FCE, 2007.

Rama, Ángel. "Medio siglo de narrativa latinoamericana (1922-1972)".

La novela en América Latina. Panoramas 1920-1980. Instituto Colombiano de Cultura, 1982. 99-202.

Rodríguez Monegal, Emir. "Borges: Una teoría de la literatura fantástica”. Revista Iberoamericana 95 (1976): 177-189.

Sarlo, Beatriz. "Borges en Sur: un episodio del formalismo criollo". Punto de Vista 16 (1982): 3-6.

. Borges, un escritor en las orillas. Siglo XXI, 2007.

- "Una poética de la ficción". Historia crítica de la literatura argentina. El oficio se afirma. Ed. Sylvia Saítta. Vol. IX. Buenos Aires: Emecé, 2004. 19-38.

Sarlo, Beatriz y Carlos Altamirano. Ensayos argentinos. De Sarmiento a la vanguardia. Ariel, 1997. 